\title{
An Evaluation of the Effectiveness of Phone Calls in Recruitment for Convalescent Plasma Donation During the COVID-19 Pandemic
}

\author{
Connor Evins ${ }^{1}$, Prerana Roth ${ }^{2}$, Jeffery Edenfield ${ }^{2}$, Xiyan Tan ${ }^{3}$, Nabihah Kumte ${ }^{1}$, Cameron Keramati ${ }^{1}$, \\ Christine MG Schammel ${ }^{4}$ and Robert Rainer ${ }^{4 *}$ \\ ${ }^{1}$ University of SC, School of Medicine Greenville, USA \\ ${ }^{2}$ Prisma Health Upstate, Department of Medicine, USA \\ ${ }^{3}$ Clemson University, School of Mathematical and Statistical Sciences, USA \\ ${ }^{4}$ Pathology Associates, USA
}

\begin{abstract}
Purpose of Study: During the COVID-19 pandemic, the FDA granted emergency use of Convalescent Plasma (CCP) as a treatment modality. Blood centers and hospitals began recruiting eligible donors, as demand exceeded supply. In the setting of an emergency, this study assessed the effectiveness of phone calls versus mailers for plasma donation recruitment.
\end{abstract}

Methods: From May to July 2019, patients that tested positive by PCR for Covid-19 were contacted to donate CCP. All patients were passively mailed a letter informing the recipient of the ability to donate CCP, as well as the need for this donation. The second group was also contacted via telephone. The callers recorded whether or not they made contact with the potential donor over the phone. Donation rates were measured for both groups and compared using a simple T-test. The phone call group was further assessed to determine the significance of donation rates between patients contacted and non-contacted.

Results: A total of 1,179 potential donors were contacted (715 phone cohort, 464 mail only cohort). Conversion rates were $6.90 \%$ for the mail group and $3.92 \%$ for the phone group. The students confirmed contact with 245 (34.27\%) of the patients, confirmed no contact with 303 (42.38\%) and did not record contact with 167 (23.36\%). Of patients who answered the phone calls, 12 (4.90\%) donated, while $233(95.10 \%)$ did not donate.

Conclusion: Conversion of potential blood donors remains a challenge. Despite the need during the pandemic, recruitment of donors was a challenge, with no statistical difference between active phone calls and passive mailers.

KEYWORDS: Convalescent plasma; Donation; Donor phone call; Recruitment; Covid-19; Pandemic

\section{INTRODUCTION}

SARS-CoV-2 is a highly pathogenic human coronavirus that has caused the Covid-19 pandemic with over 31 million cases diagnosed in the United States alone as April 2021 [1]. This very small single- stranded RNA virus is believed to have originated in Wuhan, China in a wet animal wholesale market, although exact origin is unclear [2]. The disease can have a latency period of several days before
Quick Response Code:

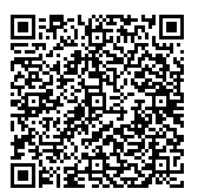

Address for correspondence: Robert Rainer, MD, Pathology Associates, USA

Received: October 01, $2021 \quad$ Published: October 21, 2021

How to cite this article: Connor E, Prerana R, Jeffery E, Xiyan T, Nabihah K, Cameron K, Schammel CM, Robert R. An Evaluation of the Effectiveness of Phone Calls in Recruitment for Convalescent Plasma Donation During the COVID-19 Pandemic. 2021- 3(5) OAJBS.ID.000338. DOI: $10.38125 / \mathrm{OAJBS} .000338$ 
presenting with a variety of nonspecific symptoms including fever, cough, headache, and in some severe respiratory failure. The severity of the illness is coupled with a high rate of transmission and pathogenicity via respiratory droplets even in asymptomatic carriers [3]. Despite significant funding and worldwide research, a year after the first reported cases treatment options remain limited. The most effective means of stopping the virus is prevention through social distancing and use of masks, good hygiene practices, and quarantining if symptomatic.

Early in the pandemic, administration of convalescent plasma obtained from persons recovered from Covid-19 infection was a primary treatment option. This was based on historical efficacy in the treatment of similar viral illnesses and its use was associated with few serious side effects [4]. Research regarding its efficacy to treat Sars-CoV-2 has been ongoing and convalescent plasma is still offered to many patients as a treatment modality.4 As the number of cases has continued to rise across the world, the supply of convalescent plasma has not been able to meet the demand [5].

Strategies to improve blood donation have been a source of debate and study. With respect to plasma donations, telephone outreach from persons knowledgeable about the process have found significant efficacy, especially for recruiting first time donors [6]. Other studies on participation found phone calls to be superior to mail-in requests, and furthermore found 3 phone calls per patient to be the maximum number to generate meaningful benefit $[7,8]$. Germain and colleagues combined phone calls with email reminders and demonstrated a minor increase in blood donation compared to phone calls alone [9]. However, this same study suggests it is not the combination of media that improves participation, but the follow up after initial contact that is important.

Another frequently observed aspect of blood donation is a massive influx of first-time donors during a national emergency. This has been extensively studied and has been shown to have potentially detrimental effects as blood supplies in the months that followed the disaster dropped substantially $[10,11]$. Regardless of this unintended consequence, it is possible that the Covid-19 pandemic would be similarly motivating compared with a natural disaster or terrorist attack. If the general public were to recognize the overwhelming need for convalescent plasma donations in the setting of a global pandemic, perhaps it would encourage more donations.

The Centers for Disease Control (CDC) and the World Health Organization (WHO) have initiated national media campaigns to encourage convalescent plasma donation. Subsequently, our single institution assembled a team of knowledgeable medical student volunteers to call patients recovered from SARS-CoV-2 between April 2020 until July 2020 in order to give them information on plasma donation. The single institution also organized a less time intensive campaign involving passive mailings to another cohort of recovered patients in the same population. We then studied the effectiveness of the phone calls to elicit donation as opposed to the mailed letters. The purpose was to determine if the time intensive phone calls were more successful with respect to donation rates in the setting of the Covid-19 pandemic [12,13].

\section{METHODS}

Following IRB approval, medical students (years 1-4) from a single medical school affiliated with the single institution were recruited to make phone contact from a list of patients who had tested positive and recovered from Covid-19 infection in May 2020. Patients were called at the primary phone number listed in the electronic medical record. The total number of patients called per student varied. Patients were required to verify their identity prior to communication about their personal medical history to ensure HIPAA compliance. Patients under 18 were excluded from the study, as were patients who were unable to communicate in English as interpreters were not readily available.

Students were required to review information provided by the single institution on the potential utility of convalescent plasma and the donation process and were provided an example script to be read to the patient during the phone call. If the patient answered, the connection with the patient was documented. If the patient did not answer, they were called two more times for a total of three phone calls. The calls were all made on different days to increase the likelihood of successfully reaching the patients. Callers left messages with all patients they did not speak with and requested a call back. If connection was unsuccessful on all 3 attempts and the student did not receive a call back despite leaving voice messages, this was documented. At least four months after the phone calls were made, a list of plasma donors from the regional blood bank was used to evaluate donor rates.

All COVID recovered patients in the study received mailed letters from the single institution encouraging plasma donation; these patients were also exposed to the generic regional and national media campaigns to encourage plasma donations during the pandemic. To evaluate the effect of phone solicitation for donation, the called cohort was compared to COVID recovered patients that did not receive a phone call but did get the letter regarding plasma donation.

Data from all called patients were stratified for age and gender and analyzed to evaluate donation rates for patients who were known to connect with the student caller and those who were known to not connect with the student caller. Data from patients that received letters only were not stratified. All data were analyzed for overall donation rate.

Statistical analyses were performed, with continuous variables reported as mean \pm standard deviation with median and interquartile range (IQR). Discrete variables were reported as $\mathrm{N}(\%)$. The $\mathrm{p}<0.05$ was considered statistically significant. Continuous variables were analyzed using T-test or Wilcoxon ranksum, while discrete variables were analyzed using Chi-square or Fisher's exact test. All analyses are carried out using R statistical software (R Foundation for Statistical Computing, version 4.0.4).

\section{RESULTS}

Overall, total of 715 patients were called by the medical students to encourage donation. Of these, $43 \%(n=304)$ were male and $57 \%$ were female $(n=411)$; the mean age of the cohort was 32.95 (Table 1). The students confirmed phone contact with 245 (34.27\%) of the called patients; no phone contact was confirmed for 303 $(42.38 \%)$ patients and there was no verification of contact for 167 $(23.36 \%)$ patients. Overall, $28(3.92 \%)$ of the patients in the total cohort donated plasma, while 687 (96.08\%) did not donate plasma (Table 1). Of those that were connected by phone, $4.9 \%$ donated plasma $(n=12)$. Interestingly, the cohort of patients who received mailed letters only was 464 patients, of which 32 (6.90\%) donated plasma (Table 1). 
Table 1: Demographics.

\begin{tabular}{|c|c|}
\hline & $\mathrm{n}=715(\%)$ \\
\hline \multicolumn{2}{|c|}{ Gender } \\
\hline Male & $304(42.52)$ \\
\hline Female & $411(57.48)$ \\
\hline \multicolumn{2}{|c|}{ Age } \\
\hline Mean \pm SD & $32.95 \pm 13.22$ \\
\hline Median (IQR) & $27(24,42)$ \\
\hline \multicolumn{2}{|c|}{ Connected } \\
\hline Yes & 245 (34.27) \\
\hline No & $303(42.38)$ \\
\hline Unknown & $167(23.36) \dagger$ \\
\hline \multicolumn{2}{|c|}{ Donated Overall } \\
\hline Yes & $28(3.92)$ \\
\hline No & $687(96.08)$ \\
\hline Donated Connected & $\mathrm{n}=245$ \\
\hline Yes & $12(4.9)$ \\
\hline No & $233(95.1)$ \\
\hline Only Received Mailed Letters* & $\mathrm{n}=464$ \\
\hline Donated & $32(6.90)$ \\
\hline Not donated & $432(93.10)$ \\
\hline
\end{tabular}

* Patients that received letters only were a separate cohort used for comparison; tdue to the high call volume and volunteer base, documentation of connected status was not achieved for all patients.

The demographic that donated plasma was evaluated and compared to those that did not donate (Table 2). Overall, 18 $(64.92 \%)$ were female and $10(35.71 \%)$ were male; this was not statistically different than those that did not donate $(p=0.56)$. The average age of those who donated was 37.79 compared to 32.75 who did not donate (Table 2 ); this also was not significantly different $(p=0.108)$.

For patients who connected with a medical student by phone, $12(4.90 \%)$ donated plasma and 233 (95.10\%) did not donate (Table 2). While gender was not statistically different between those that donated and those that did not $(p=0.554)$, age was significantly different ( $p=0.0125)$ with the mean age of those that donated 25 years, and those that did not donate 28 years (Table 2). Interestingly, for those that did not connect with the student callers, five (1.65\%) donated and 298 (98.35\%) did not donate plasma (Table 2) gender was not significantly different $(p=0.395)$ between the groups but mean age was (donated 39.4 versus not donated 28.2; $p=0.034$ ).

The donation percentages between those with whom connection was made and those that were not connected was significantly different ( $4.9 \%$ versus $1.65 \%$; $p=0.0293$ ). Similarly, the percentage of those who received mailed letters only compared with those for whom connection was not made was also statistically significant (6.9\% versus $1.65 \% ; p=0.0009$ ). When evaluating those of whom connection was made to those that received mailers only, the donation percentages were not significantly different $(4.9 \%$ versus 6.9\%; $p=0.2943$; data not shown).

Table 2: Donated vs. Not Donated (all patients).

\begin{tabular}{|c|c|c|c|c|}
\hline & & Donated & Not Donated & p-value \\
\hline \multicolumn{5}{|c|}{ All Patients ( $n=715)$} \\
\hline Gender, n(\%) & & $28(3.92)$ & 687 (96.08) & \multirow{3}{*}{0.56} \\
\hline Male & $304(42.52)$ & $10(35.71)$ & $294(42.79)$ & \\
\hline Female & $411(57.48)$ & $18(64.29)$ & 393 (57.21) & \\
\hline Age, Mean \pm SD & & $37.79 \pm 15.84$ & $32.75 \pm 13.08$ & 0.108 \\
\hline Median (IQR) & & $31.5(23.75,52)$ & $27(24,41.5)$ & 0.172 \\
\hline \multicolumn{5}{|c|}{ Connected patients $(n=245)$} \\
\hline Gender, n(\%) & & $12(4.90)$ & 233 (95.10) & \multirow{3}{*}{0.554} \\
\hline Male & $112(45.71)$ & $4(33.33)$ & $108(44.08)$ & \\
\hline Female & $133(54.29)$ & 8 (66.67) & 125 (51.02) & \\
\hline Age, Mean \pm SD & & $25 \pm 3.33$ & $28 \pm 7.37$ & 0.0125 \\
\hline Median (IQR) & & $24.5(23,25)$ & $25(23,30)$ & 0.193 \\
\hline \multicolumn{5}{|c|}{ Not connected patients $(n=303)$} \\
\hline Gender, n (\%) & & $5(1.65)$ & 298 (98.35) & \multirow{3}{*}{0.395} \\
\hline Male & $122(40.26)$ & $3(60)$ & 119 (39.93) & \\
\hline Female & $181(59.74)$ & $2(40)$ & $179(60.07)$ & \\
\hline Age, Mean \pm SD & & $39.4 \pm 7.99$ & $28.16 \pm 7.66$ & 0.034 \\
\hline Median (IQR) & & $42(33,42)$ & $27(23,30)$ & 0.0035 \\
\hline
\end{tabular}




\section{DISCUSSION}

Despite evidence that phone calls were an effective strategy for plasma donations and that national disasters historically have increased blood donations $[6,10,11]$ medical student phone calls to patients recovered from Covid-19 infection did not appreciably increase convalescent plasma donations. Overall, for the entire cohort $(n=715)$, only $4 \%(n=28)$ of recovered COVID patients donated plasma. This group includes those for whom connection was made by phone and those with whom connection was not made.

Within the group with whom connection was made $34.27 \%$ of the cohort), with the idea that calling and communicating directly with patients would promote donation, the overall donation rate was only $4.90 \%$, not significantly different from the rate for the entire group, suggesting that direct connection only had a minor positive impact in the donation effort. In fact, the effort to directly communicate by calling repeatedly and leaving a message appeared detrimental to the donation effort as this group had a donation rate of only $1.65 \%$ (Figure 1). Given the fact that all patients in the study were receiving mailed letters encouraging plasma donation as well as being exposed to public service marketing encouraging donation, the group that received the multiple calls and messages could have had 'information overload', causing them to retract from participation as the input about plasma donation exceeded the processing capacity to actually donate. [Speier] This theory could be further supported when evaluating the patients who received mailed letters only along with the general public service marketing; this cohort donated at a rate of $6.9 \%$ (Figure 1).

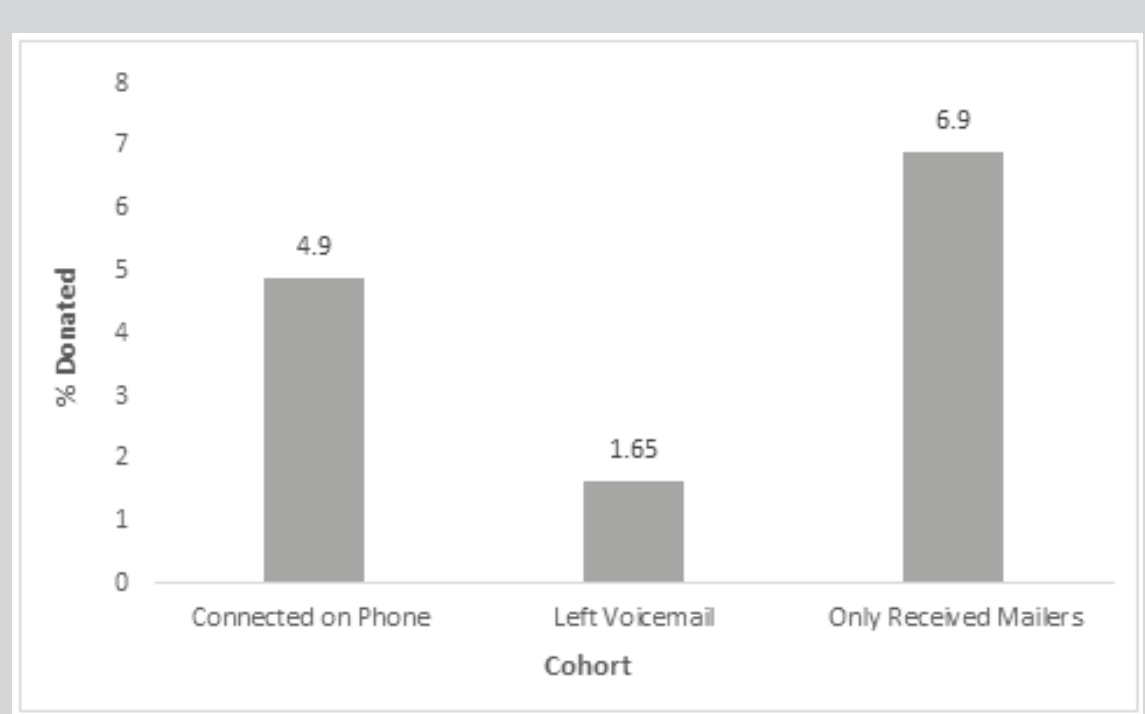

Figure 1: Percentages of donors who connected on phone, received voicemail only, or received mailers only.

In evaluating other factors that may have influenced the donation rate, while gender did not predict donation overall or in any cohort (Table 2), age was found to be significantly different for those who donated within both the connected cohort and the not connected cohorts. Within the connected cohort, the mean age of those that donated was 25 years, while the mean age of those that did not donate was 28 years (Table 2). Within the cohort that did not directly connect (multiple calls and messages), the mean age of those that donated was older (39 years) compared to those that did not (28 years). These data may reflect the complicated relationship that individuals have with phone calls and the changing etiquette around communication which emphasizes the preference of texting over voice calls for certain demographics ( $>20$ ). [Forgays] Larger more sophisticated studies would need to be done to ascertain the optimal communication for specific age groups to maximize connection and induce motivation.

Overall, the data suggests that, between direct patient communication by phone, multiple attempts at direct communication and leaving messages and direct mail, mail appears to be the superior means of encouraging donation. Although not statistically significant $(p=0.2943)$ the donation rate was higher for those that received the letters only versus direct connection by phone. Given the fact that the patients in the phone call cohort also received the letters and general public service marketing, and yet no increase in donation was noted, the time intensive process of calling people to encourage plasma donation may not be efficacious.
Making individual phone calls is a time-consuming task with an overall low yield in our experience. Given that we were unable to even connect with $41.6 \%$ of patients through phone calls speaks to the fact that perhaps other means such as email or texting would be more effective. It is unclear why those who did discuss plasma donation with a student did not donate more frequently. One consideration could be the impact of the pandemic and the attendant national lockdown and associated restrictions limited patient resources and willingness to travel for donation. Another is the patient population may have been wary of exposing themselves to the disease again after a potentially difficult recovery. While the COVID-19 pandemic was certainly a national emergency throughout 2020 , the type of emergency was quite different when compared to natural disasters or terror attacks that have been previously studied with respect to blood donation rates. While those events involved significant trauma and clear evidence of injury, COVID-19 infections were likely viewed with more of a subtlety versus pertaining as a blood product necessity for community wellness.

The suggestion that, perhaps, the overall low donation rates could simply reflect an education deficit on the need for convalescent plasma seems unlikely as connecting with patients for one-on-one phone conversations to address the need and benefits did not improve donation rates. Additionally, the public service marketing was pervasive making educational material ubiquitously available. Overall, these data study suggest direct patient connection through phone calls are of limited effectiveness in motivating 
plasma donations from COVID recovered patients. While mailed letters had a greater donation rate, overall responsiveness to the request for donation was low. Factors such as fear, over saturation of information and other factors should be evaluated to determine optimal strategies to motivate individuals in times of healthcare crises.

\section{CONTRIBUTORSHIP STATEMENT}

CE, PR, JE, and RR worked together to plan the study, develop the protocols, and coordinate with the phone call volunteers. CE, $\mathrm{CK}$, and NK made phone calls to potential donors, collected and organized the data, and helped with some of the data analysis. XT analyzed the data and created the tables. CE wrote the paper and CMGS edited and submitted the manuscript and serves as the guarantor of the content. All authors read and edited the paper before submission.

\section{ACKNOWLEDGMENT}

The authors would like to thank the volunteer department at U of SC School of Medicine Greenville for helping secure students to make phone calls to increase plasma donations. They would also like to thank the Blood Connection (C) for working with Prisma Health Upstate and finding and securing potential donors for this project.

\section{REFERENCES}

1. COVID-19 dashboard by the center for systems science and engineering (CSSE) at Johns Hopkins University.

2. Ouassou H, Kharchoufa L, Bouhrim M, Daoudi NE, Imtara H, et al. (2020) The pathogenesis of coronavirus disease 2019 (COVID-19): evaluation and prevention. J Immunol Res 2020: 1357983.
3. Han Y, Yang H (2020) The transmission and diagnosis of 2019 novel coronavirus infection disease (COVID-19): a Chinese perspective. J Med Virol 92(6): 639-644

4. Chen L, Xiong J, Bao L, Shi Y (2020) Convalescent plasma as a potential therapy for COVID-19. The Lancet Infect Dis 20(4): 398-400.

5. Hartmann J, Klein HG (2020) Supply and demand for plasma-derived medicinal products - A critical reassessment amid the COVID-19 pandemic. Transfusion 60(11): 2748-2752.

6. Thorpe R, Masser BM, Nguyen L, Gemelli CN, Davison TE (2020) Bringing new plasma donors back: testing the efficacy of telephone and e-mail communications with first-time donors. Transfusion 60(7): 1463-1469.

7. Siemiatycki J (1979) A comparison of mail, telephone and home interview strategies for household health surveys. Am J Public Health 69(3): 238-245.

8. Iqbal R, Haroon A, Jabbar A, Babar N, Qureshi R (2012) What method of contact works best for recruiting participants in a study: lessons for health care researchers? J Pak Med Assoc 62(12): 1293-1297.

9. Germain M, Godin G (2016) The relative efficacy of telephone and email reminders to elicit blood donation. Vox Sang 110(3): 278-281.

10. Busch MP, Guiltinan A, Skettino S, Cordell R, Zeger G, et al. (1991) Safety of blood donations following a natural disaster. Transfusion 31(8): 719723.

11. Glynn SA, Busch MP, Schreiber GB, et al. (2003) Effect of a national disaster on blood supply and safety: The September 11 experience. JAMA 289(17): 2246-2253.

12. Speier C, Valacich JS, Vessey I (1999) The influence of task interruption on individual decision making: An information overload perspective. Decision Sciences. 30 (2): 337-360.

13. Forgays DK, Hyman I, Schreiber J (2014) Texting everywhere for everything: gender and age differences in cell phone etiquette and use. Comp Hum Beh 31: 314-321. 